\title{
Aging of rat retina: transcriptome study
}

\author{
O.S. Kozhevnikova*, D.V. Telegina, N.G. Kolosova \\ Institute of Cytology and Genetics SB RAS, Novosibirsk, Russia \\ *e-mail: oidopova@bionet.nsc.ru
}

Key words: aging, retinopathy, senescent-accelerated OXYS rats

Motivation and Aim: The aging process is the major risk factor for age-related diseases, including age-related macular degeneration (AMD). Age-related macular degeneration (AMD) is a complex disease leading to visual dysfunction through a variety of mechanisms and complications. The senescence-accelerated OXYS rats develop retinopathy with clinical and morphological manifestations similar to AMD. Clinical, histological and molecular manifestations of retinopathy in OXYS rats, the results of identification quantitative trait loci (QTLs) and transcriptome studies will be presented. OXYS rats first demonstrated fundoscopic changes at 1.5 months of age with the appearance of atrophic areas in the RPE and choriocapillaris. Older rats developed thickening of Bruch's membrane, drusen and RPE detachments. By 12 months, some animals demonstrated photoreceptor atrophy, decreased ERGs, destruction of the choriocapillaris with fibrosis and in some cases hemorrhagic detachment of the retina due to neovascularization.

Methods: In order to identify the impact of aging process on in response to normal aging and progression of AMD-like retinopathy, we compared gene expression profiles of retina from young and old OXYS and control Wistar rats by RNA sequencing (RNA-Seq).

Results: The majority of DE genes are related to the immune system and extracellular matrix turnover. Little age-regulated genes were common for the two strains, suggestive of different rates and mechanisms of aging. Hundreds genes showed significant differences in expression between the two strains. These genes are involved in disease-associated pathways such as immune response, inflammation, apoptosis, $\mathrm{Ca}^{2+}$ homeostasis, and oxidative stress. We can conclude that the development of retinopathy in OXYS rats is associated with an imbalance in immune and inflammatory responses. Aging had significant effects on the expression of inflammatory genes but their composition was different in the retina of OXYS and Wistar rats. The retinopathy development in OXYS rats accompanied by downregulation of immune response genes in the retina. This indicates that any disturbances in immune defenses can accompany retinal disease, not only upregulation, but also downregulation, which can be explained within the framework of immunosenescence theory. Our data support the view that the genetic background has a profound impact on AMD development.

Acknowledgements: Supported by the RSF No. 18-75-00031. 